\title{
NONLINEAR FRICTION ESTIMATION FOR DIGITAL CONTROL OF DIRECT-DRIVE MANIPULATORS
}

\author{
B. Bona, M. Indri, N. Smaldone \\ Dipartimento di Automatica e Informatica, Politecnico di Torino \\ Corso Duca degli Abruzzi, 24, 10129 Torino, Italy \\ phone: +390115647066 \\ fax: +39011564 7099 \\ e-mail: basilio.bona@polito.it,marina.indri@polito.it,nicola.smaldone@polito.it
}

Keywords: Friction, stiction, model identification, direct-drive manipulator, experimental validation.

\begin{abstract}
Control algorithms for precise motion control require a complete robot dynamic compensation, including also nonlinear friction phenomena occurring at very low velocities. Several models, including both static and dynamic friction, have been proposed in the last few years, the most used being the LuGre model and its modifications. Unfortunately, a good estimation of the dynamic friction parameters is often quite difficult to be achieved in practice. In this paper, the parameters of the static part of this friction model are estimated for a doublearm direct-drive planar manipulator, and the identified model is used for torque reconstruction during an assigned motion, by considering the nominal values of the robot inertial parameters. The results are compared with those obtained by estimating the manipulator inertial parameters, together with the parameters of a simplified third order polynomial friction model. Experiments confirm the validity of the available, nominal, inertial values, and show that, at least in our case, similar results are obtained by the two friction models.
\end{abstract}

\section{Introduction}

Friction torques are among the principal phenomena affecting the performances of motion control algorithms at low velocities, as they can lead to significant tracking errors, stick-andslip motion and limit cycles [14, 3, 5, 12].

The nonlinear nature of friction torques makes simple PID controllers questionable, especially when the control algorithm must cope with both low velocity stiction torques and high velocity coupling torques between links $[11,5,16,17,2,19]$.

This paper presents and discusses the results of parameter identification experiments performed on a direct-drive double arm planar manipulator, having noticeable nonlinear friction torques on both joints. The friction is assumed to be described by the so-called LuGre model, one of the most accepted friction models available in literature $[11,4,12,10]$.

A DSP-based real-time architecture, implementing simple joint-independent PD control laws, is used to collect the data necessary to reconstruct the friction torques and to simulate the manipulator dynamic behavior for the validation of the developed friction model. Finally, the results are compared with those obtained by estimating the manipulator inertial parameters, together with the parameters of a simplified third order polynomial friction model. Experiments confirm the validity of the available, nominal, inertial values, and show that, at least in our case, similar results are obtained by the two friction models.

The outline of the paper is as follows. Section 2 is devoted to friction modelling and identification, while in Section 3 a brief description of the manipulator experimental setup is given. Section 4 describes the dynamic and friction model identification procedure, reports the experimental results for validation and draws the conclusions.

\section{Friction modelling}

In this section, the main characteristics of the friction phenomena and the various friction models proposed in literature are briefly reviewed, before discussing the experimental results obtained in the friction identification tests, performed on the considered robot.

Different models have been proposed in literature, see e.g. $[3,11,4,12,13,20,15,9]$ to describe in a more or less accurate way all the friction components. A basic classification of the friction models is relative to their static or dynamic characteristics [18].

The classical static models describe friction as a function of the relative velocity of the bodies in contact, taking into account some of the various aspects of the friction force, such as Coulomb friction, viscous friction, stiction, and the so-called Stribeck effect, which is relative to the low-velocities region, in which friction decreases as velocity increases. Different nonlinear functions have been proposed to describe such aspects, taking into account only the current velocity value (thus defining static friction models). The simplest choice is given by a polynomial function of a sufficiently high order.

The model proposed in [4] can be considered as an intermediate step towards dynamic models; it introduces temporal dependencies for stiction and the Stribeck effect, but it does not handle presliding displacement, which is addressed by a proper 
dynamic friction model dealing with the behavior of the microscopical contact points between the surfaces.

The well-known LuGre model [12, 18] takes into account both the steady-state friction curve and the presliding phase by means of flexible bristles, representing the contact points of the moving surfaces. According to such a model, the behavior of the friction torque $\tau_{f i}$ on the $i$-th joint of a manipulator can be described by the following equations:

$$
\begin{aligned}
\dot{z}_{i} & =\dot{q}_{i}-\frac{\left|\dot{q}_{i}\right|}{g_{i}\left(\dot{q}_{i}\right)} \sigma_{0 i} z_{i} \\
\tau_{f i} & =\sigma_{0 i} z_{i}+\sigma_{1 i} \dot{z}_{i}+f_{i}\left(\dot{q}_{i}\right)
\end{aligned}
$$

where $\dot{q}_{i}$ is the angular velocity of the joint ( $q_{i}$ being the joint position coordinate), $z_{i}$ is a state variable representing the average bristle deflection for joint $i, \sigma_{0 i}$ and $\sigma_{1 i}$ are model parameters assumed to be constant, and $g_{i}\left(\dot{q}_{i}\right)$ and $f_{i}\left(\dot{q}_{i}\right)$ model the Stribeck effect and the viscous friction, respectively. For constant velocity, the steady-state friction torque is then given by:

$$
\tau_{f i_{s s}}=g_{i}\left(\dot{q}_{i}\right) \operatorname{sgn}\left(\dot{q}_{i}\right)+f_{i}\left(\dot{q}_{i}\right)
$$

Different parameterizations are possible for functions $g_{i}\left(\dot{q}_{i}\right)$ and $f_{i}\left(\dot{q}_{i}\right)$ : the first one is a nonlinear function of velocity, generally expressed by means of exponential terms, while the second one can be given by a simple linear viscous function or by a higher order polynomial function, when required for a better fitting with the collected experimental data.

\section{The Experimental Setup}

The considered robot is a planar two-arms manipulator, manufactured by IMI (USA), and sketched in Figure 1. The maximum extension of the links $\left(\ell_{1}+\ell_{2}\right)$ is about $0.7 \mathrm{~m}$, the angular limits being \pm 2.15 rad for both joints.

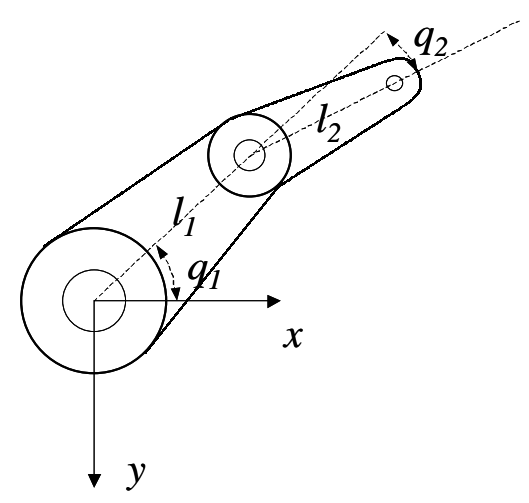

Figure 1: The geometrical scheme of the IMI planar manipulator.

The arms are driven by a couple of brushless NSK Megatorque direct drives. Two control modes are available, the Torque Mode and the Velocity Mode: on the basis of the resolver signals, a current loop is closed to regulate the torque in the first case, whereas a further velocity loop is added in the second mode. The basic mode is the Torque Mode, and it will be the only one used in this work to control the manipulator.

The control architecture, called OpenDSP, has been developed by the Mechatronics Laboratory of the Politecnico di Torino both in its software and hardware parts, and represents the enhanced, industrial evolution of an older, educational version, illustrated in [1]. OpenDSP consists of a DSP board and a programmable input/output board. The system is linked via enhanced parallel port (EPP) protocol to a host PC, and by some connections to each axes interface. A Matlab environment with Simulink runs on the host PC and can interact with the DSP board. The architecture of the complete experimental setup is sketched in Figure 2.

The OpenDSP system includes a new toolbox for Matlab called MatDSP, which allows the Matlab-code interaction with the DSP. In this way it is possible to read or change any variable processed by the DSP, in synchronous or asynchronous mode, and the control algorithms written in $\mathrm{C}$ can be compiled, downloaded and started/paused on DSP.

More details about the OpenDSP architecture and real-time software, which belongs to the so-called round-robin with interrupts architecture group, can be found in [6, 7].

\section{Dynamic and Friction Model Identification}

The model of the manipulator under study can be described by the following second-order nonlinear differential equation in $\mathbb{R}^{2}$ :

$$
\boldsymbol{M}(\boldsymbol{q}) \ddot{\boldsymbol{q}}+\boldsymbol{C}(\boldsymbol{q}, \dot{\boldsymbol{q}}) \dot{\boldsymbol{q}}+\boldsymbol{\tau}_{f}(\boldsymbol{q}, \dot{\boldsymbol{q}})=\boldsymbol{\tau}_{c}
$$

where $\boldsymbol{q}, \dot{\boldsymbol{q}}$, and $\ddot{\boldsymbol{q}}$ are the vectors of joint angles, angular velocities and angular accelerations, $\boldsymbol{M}(\boldsymbol{q})$ is the configurationdependent inertia matrix, including both links and motors inertia, $\boldsymbol{C}(\boldsymbol{q}, \dot{\boldsymbol{q}}) \dot{\boldsymbol{q}}$ is the term containing Coriolis and centrifugal torques, $\boldsymbol{\tau}_{f}$ is the friction torque vector, and $\tau_{c}$ is the command torque vector. The gravitation effects are considered to be negligible, due to the physical placement of the manipulator arms moving in the horizontal plane. Each command torque can be expressed as a function of the command input voltage $v_{m}$ of the corresponding actuator according to equation

$$
\tau_{c}=K_{v \tau} v_{m}
$$

where $K_{v \tau}$ is the voltage to torque gain.

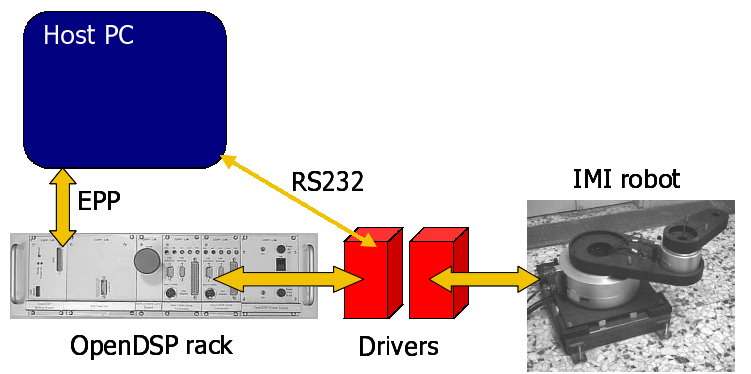

Figure 2: The experimental setup. 
In a previous paper [8], friction has been characterized taking into account only two main aspects of that phenomenon, i.e. Coulomb friction and viscous friction, obtaining only partially satisfying results. This paper investigates the possibility of obtaining a more accurate reconstruction of the real behavior of the friction torques. Only static friction is considered at this moment.

Two models are considered to represent the friction torques $\tau_{f i}$ on each joint:

a) the static part of the LuGre model;

b) a third-order polynomial function of $\dot{q}_{i}$.

Besides, while the available, nominal values of the robot inertial parameters are used in the experiments performed to estimate the parameters of the LuGre model, as described in Subsection 4.1, a complete robot dynamic identification is carried out when the friction polynomial model is considered, as described in Subsection 4.2.

\subsection{Parameter identification of the static LuGre friction model}

Only the static part of the LuGre model in (1) and (2) is used to represent the friction torques by considering $\dot{z}_{i}=0, i=1,2$ (thus obtaining $\tau_{f i}=\tau_{f i_{s s}}$ ), and the following expressions for the Stribeck curve $g_{i}\left(\dot{q}_{i}\right)$ and the viscous friction $f_{i}\left(\dot{q}_{i}\right)$, for $i=1,2$ :

$$
\begin{aligned}
& g_{i}\left(\dot{q}_{i}\right)=\alpha_{0 i}+\alpha_{1 i} \mathrm{e}^{-\frac{\dot{q}_{i}}{\omega_{s 1, i}} \operatorname{sgn}\left(\dot{q}_{i}\right)}+ \\
& +\alpha_{2 i}\left(1-\mathrm{e}^{-\frac{\dot{q}_{i}}{\omega_{s 2, i}} \operatorname{sgn}\left(\dot{q}_{i}\right)}\right) \\
& f\left(\dot{q}_{i}\right)=\alpha_{3 i} \dot{q}_{i}+\alpha_{4 i} \dot{q}_{i}^{2}
\end{aligned}
$$

The choice of expression (6) proposed in [11], instead of the more usual Stribeck function given in $[12,18]$, is motivated by the possibility to achieve a better data fitting in our case, when used together with the second order viscous friction function (7).

The estimation of the resulting steady-state friction curve (3) is performed by tests at different constant joint velocity values. A PD control law (with $1 \mathrm{~ms}$ sampling time and the actuators in Torque Mode), with high gains to avoid stick-slip phenomena, has been used in the experimental tests to move each joint at low velocity values. Acquisition at high velocity has been performed letting the joint rotate freely without the links, until a dynamic equilibrium situation at constant velocity is achieved.

The friction torque data have been indirectly derived in the open loop tests by considering:

$$
\boldsymbol{\tau}_{c k}=\boldsymbol{\tau}_{f k}
$$

where $\tau_{c k}$ and $\tau_{f k}$ are the $k$-th samples of the applied motor torques and of the joint friction torques, respectively. In the closed loop tests at low velocities the following relation, derived from the manipulator dynamic equation (4), has been considered:

$$
\boldsymbol{\tau}_{f}(\dot{\boldsymbol{q}})+\boldsymbol{\tau}_{e r r}=\boldsymbol{\tau}_{c}-\boldsymbol{M}(\boldsymbol{q}) \ddot{\boldsymbol{q}}-\boldsymbol{C}(\boldsymbol{q}, \dot{\boldsymbol{q}})
$$

where $\tau_{e r r}$ is a torque vector that contains all neglected modelling errors including dynamic friction phenomenon. The nominal inertial parameters values (see the next subsection for more details) have been used to compute matrices $\boldsymbol{M}(\boldsymbol{q})$ and $\boldsymbol{C}(\boldsymbol{q}, \dot{\boldsymbol{q}})$.

The parameter values to be identified in (3), with $g_{i}\left(\dot{q}_{i}\right)$ and $f_{i}\left(\dot{q}_{i}\right)$ defined as in (6) and (7), should be seven for each joint (the five $\alpha$ 's together with $\omega_{s 1, i}$ and $\omega_{s 2, i}$ ). By the observation of the acquired data, tentative values between 0.1 and $0.3 \mathrm{rad} / \mathrm{s}$ have been considered for the exponential parameters $\omega_{s 1, i}$ and $\omega_{s 2, i}$, and a least square algorithm has been applied to a linearized expression of (3)-(7) to estimate the $\alpha$ 's parameters for each joint. By some iterations, the values reported in Table 1 have been obtained.

\begin{tabular}{|c|c|c|c|c|}
\hline & $\begin{array}{c}\text { Joint 1 } \\
\omega>0\end{array}$ & $\begin{array}{c}\text { Joint 1 } \\
\omega<0\end{array}$ & $\begin{array}{c}\text { Joint 2 } \\
\omega>0\end{array}$ & $\begin{array}{c}\text { Joint 2 } \\
\omega<0\end{array}$ \\
\hline$\alpha_{0}$ & 40.854 & -46.473 & 17.837 & 3.408 \\
\hline$\alpha_{1}$ & -32.454 & 53.873 & -14.837 & -0.408 \\
\hline$\alpha_{2}$ & -31.233 & 55.738 & -14.998 & -0.635 \\
\hline$\alpha_{3}$ & -0.760 & -0.293 & -0.156 & -0.104 \\
\hline$\alpha_{4}$ & -0.262 & 0.177 & -0.050 & 0.036 \\
\hline$\omega_{s 1}$ & 0.19 & 0.14 & 0.2 & 0.3 \\
\hline$\omega_{s 2}$ & 0.17 & 0.15 & 0.19 & 0.1 \\
\hline
\end{tabular}

Table 1: Estimated static parameters of the LuGre friction model.

Figures 3 and 4 show the resulting steady-state friction torque together with the experimental data for the first joint (for positive and negative velocity values, respectively). Similar results have been obtained for the second joint.

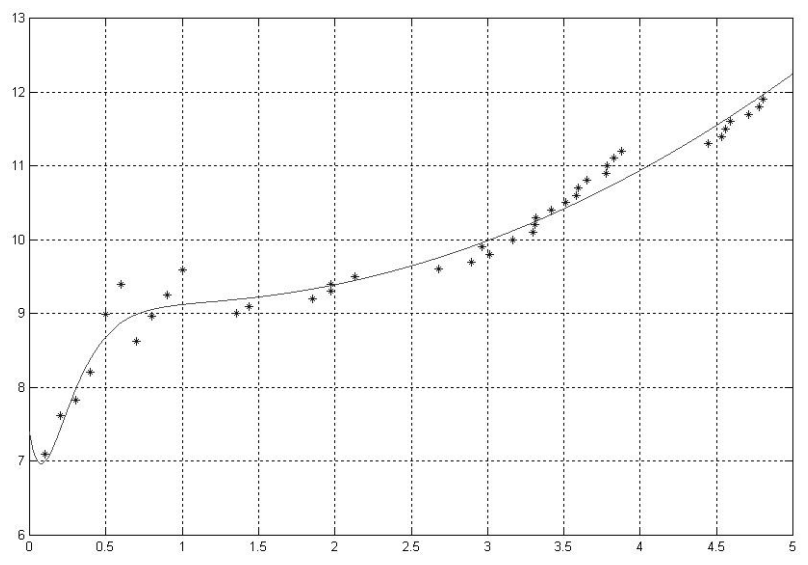

Figure 3: Friction torque (Nm) vs. positive velocity ( $\mathrm{rad} / \mathrm{s})$ on joint 1 . 


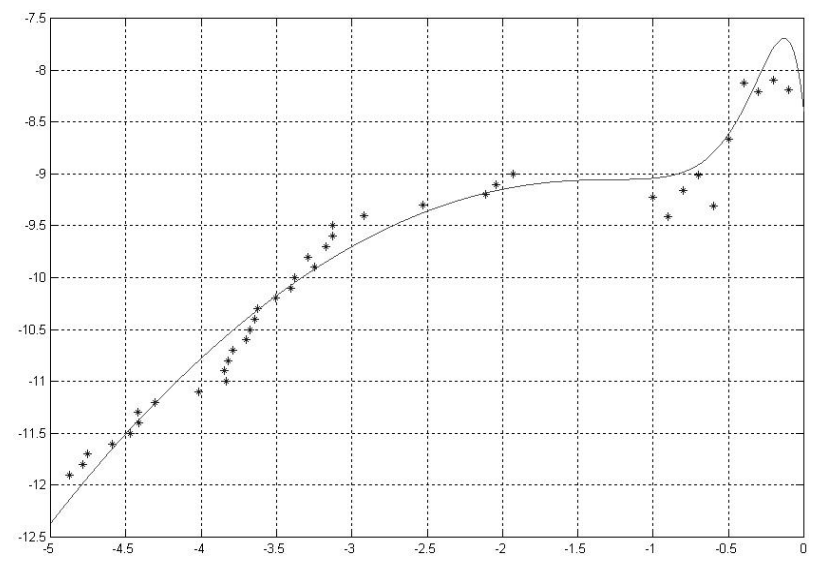

Figure 4: Friction torque (Nm) vs. negative velocity $(\mathrm{rad} / \mathrm{s})$ on joint 1 .

\subsection{Dynamic Model Identification with Polynomial Fric- tion Torques}

Let the friction torque on the $i$-th joint be described by the following third-order polynomial function:

$$
\tau_{f i}=a_{0 i} \operatorname{sign}\left(\dot{q}_{i}\right)+a_{1 i} \dot{q}_{i}+a_{2 i} \operatorname{sign}\left(\dot{q}_{i}\right) \dot{q}_{i}^{2}+a_{3 i} \dot{q}_{i}^{3}
$$

It is then possible to rewrite the manipulator dynamic model (4) in the following form:

$$
\boldsymbol{\tau}=\boldsymbol{D}(\boldsymbol{q}, \dot{\boldsymbol{q}}, \ddot{\boldsymbol{q}}) \boldsymbol{\theta}
$$

which is linear with respect to the vector $\boldsymbol{\theta}$, containing the identifiable dynamic parameters of the robot, and the friction parameters that define the friction torques (10), and $\boldsymbol{D}(\boldsymbol{q}, \dot{\boldsymbol{q}}, \ddot{\boldsymbol{q}})$ is properly defined. In particular, $\boldsymbol{\theta}$ is given by:

$$
\begin{gathered}
\boldsymbol{\theta}=\left[\begin{array}{llllllllll}
\Gamma_{1 z}+m_{2} l_{1}^{2} & m_{2} s_{2 x} & m_{2} s_{2 y} & \Gamma_{2 z} \\
a_{01} & a_{11} & a_{21} & a_{31} & a_{41} & a_{02} & a_{12} & a_{22} & a_{32} & a_{42}
\end{array}\right]^{T}
\end{gathered}
$$

i.e., the identifiable dynamic parameters are the inertia moments of the links with respect to the $z_{0}$-axis (i.e. the axis perpendicular to the motion plane) and the first order moments $m_{2} s_{2 x}, m_{2} s_{2 y}$ of the second link.

According to the method developed in [8], parameter identification has been performed by collecting data on an "optimal" trajectory (i.e. a trajectory that optimally excites the robot dynamics described by model (11)) of the following type:

$$
q_{i}(t)=\sum_{j=1}^{n_{a}} \alpha_{j, i} \sin \left(\omega_{j, i} t\right),
$$

for the $i$-th joint. In particular, the considered reference trajectory (reported in Figure 5) is a four-harmonics function, having duration time $T=15 \mathrm{~s}, \alpha_{11}=-0.4355, \alpha_{21}=-0.4032$, $\alpha_{31}=-0.5, \alpha_{41}=-0.371, \alpha_{12}=0.371, \alpha_{22}=0.2097$, $\alpha_{32}=0.4677, \alpha_{42}=0.3387, \omega_{11}=0.7213, \omega_{21}=0.8656$, $\omega_{31}=1.082, \omega_{41}=1.8754, \omega_{12}=1.8754, \omega_{22}=2.0918$,

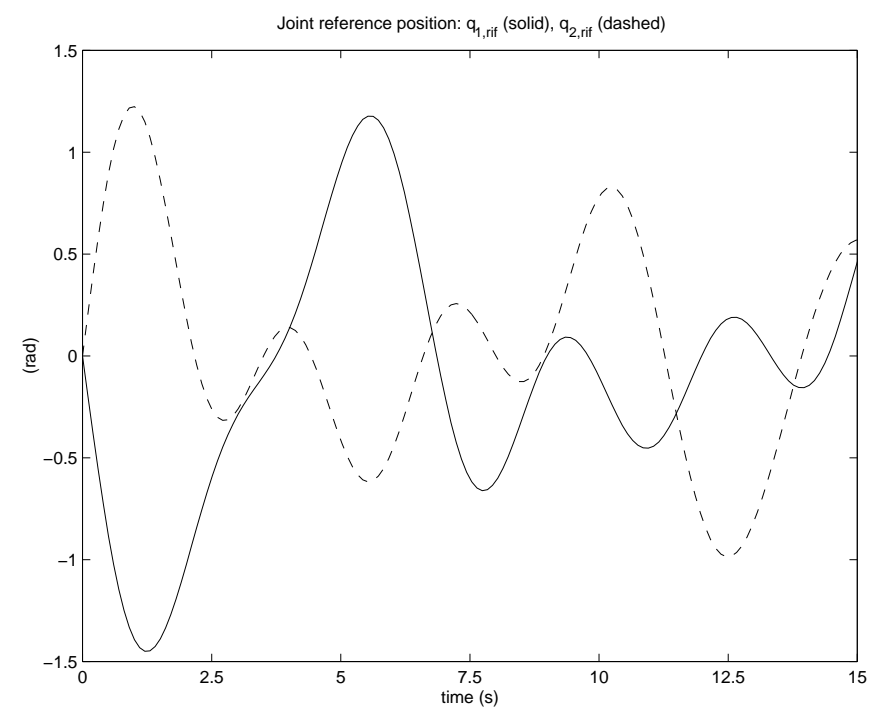

Figure 5: Joint reference trajectory for identification

$\omega_{32}=0.8656, \omega_{42}=1.3705\left(\right.$ where the $\omega_{i, j}$ terms are expressed in $\mathrm{rad} / \mathrm{s}$ ).

The same PD control law, used in the tests described in the previous subsection, has been applied to repeat the trajectory ten times, in order to investigate the repeatability of the system and the measurement noise. Joint position and torque data have been collected, whereas velocities and accelerations have been computed via software (with the insertion of a proper filtering action).

The Least-Squares algorithm described in [8] has been used for the off-line parameter estimation, collecting more than 2500 equally spaced samples for each trajectory repetition. The final obtained estimates are collected in:

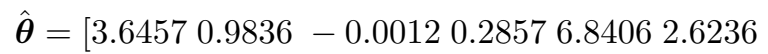

$$
\begin{aligned}
& -1.15800 .46802 .35120 .3069-0.33740 .1252]^{T}
\end{aligned}
$$

The comparison with the values obtained from the manufacturer data for the first four parameters, given by:

$$
\begin{aligned}
& \theta_{\text {nom }, 1}=3.689 \mathrm{~kg} \cdot \mathrm{m}^{2}, \theta_{\text {nom }, 2}=0.97 \mathrm{~kg} \cdot \mathrm{m}, \\
& \theta_{\text {nom }, 3}=0 \mathrm{~kg} \cdot \mathrm{m}, \theta_{\text {nom }, 4}=0.275 \mathrm{~kg} \cdot \mathrm{m}^{2},
\end{aligned}
$$

confirm the validity of the results relative to the inertial parameter estimates. The estimated parameters have been used to reconstruct the torques by the robot dynamic model (11) with $\hat{\boldsymbol{\theta}}$ instead of $\boldsymbol{\theta}$, and to compare them with the measured ones, as shown by Figure 6, with reference to the first part of one of the trajectory repetitions; torque errors are also reported in the same figure. The relative estimation errors, defined as the ratio between the rms estimation error and the rms value of the measured torques, result to be $\varepsilon_{r, 1}=31.8 \%$, and $\varepsilon_{r, 2}=31.7 \%$ for the two joints. It must be noted that local peaks of torque error correspond to changes of the velocity sign, i.e., when the used friction model is probably not adequate to well describe the present phenomena. 

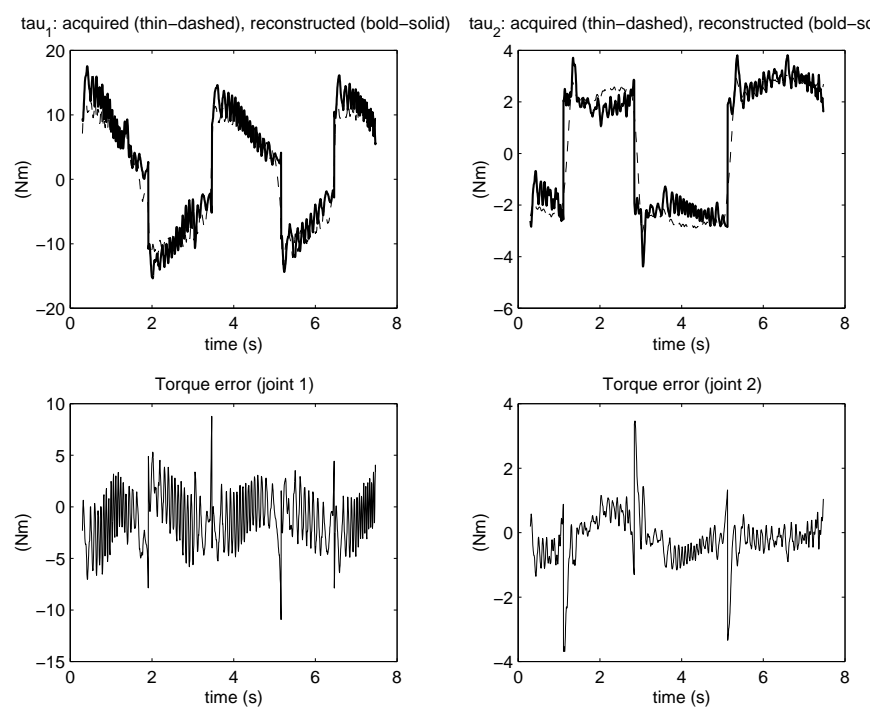

Figure 6: Torque reconstruction for the four-harmonics trajectory.

\subsection{Torque Reconstruction: Comparisons and Conclu- sions}

The results obtained by using the two different friction models have been compared by letting the manipulator execute a cartesian circular trajectory. The measured torques have been compared with the ones reconstructed by using the robot dynamic model, considering $(i)$ the nominal values of the inertial parameters together with the friction static LuGre model identified in Subsection 4.1, and (ii) the parameter vector $\hat{\boldsymbol{\theta}}$ estimated in Subsection 4.2. Figures 7 and 8 show the obtained results.
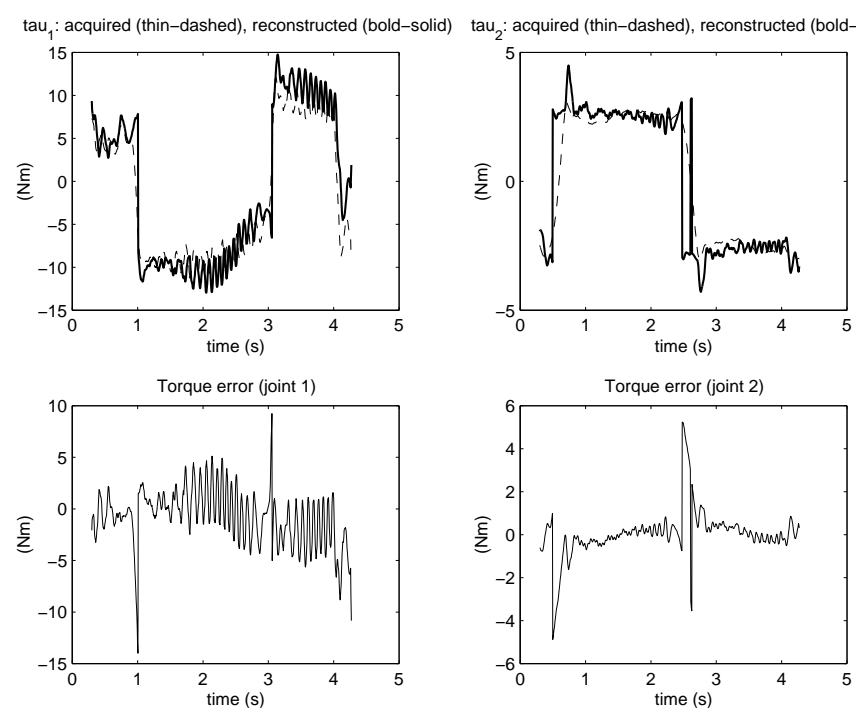

Figure 7: Torque reconstruction for the circular trajectory with the LuGre friction model.

The relative estimation errors are $\varepsilon_{r, 1}=35.8 \%, \varepsilon_{r, 2}=45.9 \%$ with the LuGre model, and $\varepsilon_{r, 1}=33.1 \%, \varepsilon_{r, 2}=39.9 \%$ with the polynomial friction model. As the figures show, the quality
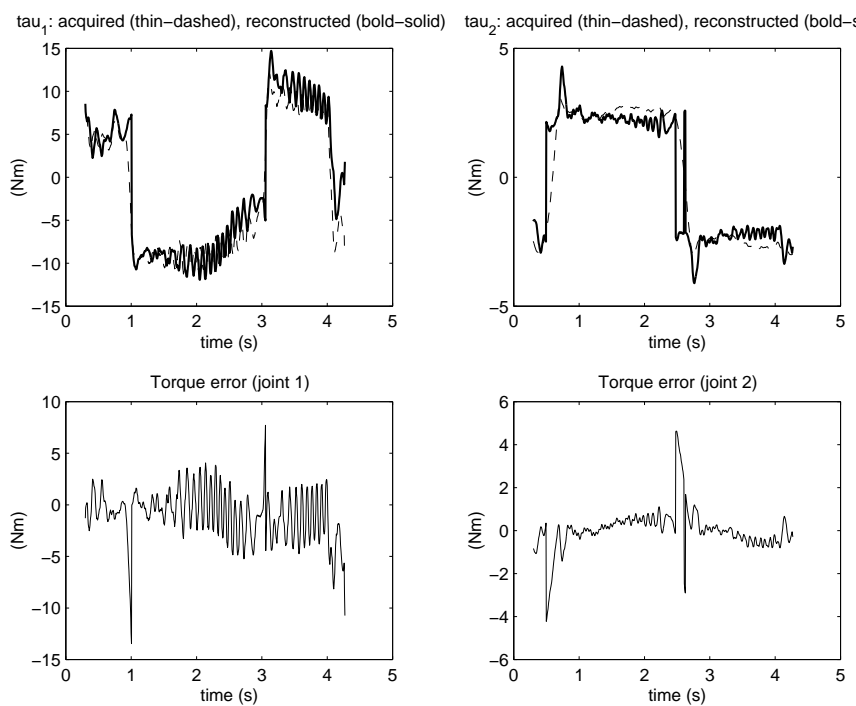

Figure 8: Torque reconstruction for the circular trajectory with the polynomial friction model.

of the torque reconstruction is comparable in the two cases: taking into account also the error peaks when velocity changes sign, the reconstruction performed in the second case seems to be a little better.

It seems then that the use of the more accurate static LuGre model gives no particular advantage in our case, if the dynamic friction component is not taken into account. Current work is devoted to the identification of this component, even if practical difficulties arise, since more accurate position measurements are required, and to the application of the identified friction models to control purposes.

\section{Acknowledgments}

This work was partially supported by Italian Ministry of University and Research (MIUR) under MISTRAL and MATRICS National Research Projects.

\section{References}

[1] A. Argondizza, B. Bona, and S. Carabelli. Automatic control teaching with matlab and matdsp. In European Control Conference (ECC 97), Brussel, July 1997.

[2] B. Armstrong, D. Neevel, and T. Kusik. New results in npid control: Tracking, integral control, friction compensation and experimental results. IEEE Trans. on Control Systems Technology, 9(2):399-406, March 2001.

[3] B. Armstrong-Hélouvry. Control of Machines with Friction. Kluwer Academic Publishers, Boston, 1991.

[4] B. Armstrong-Hélouvry, P. Dupont, and C. Ca nudas de Wit. A survey of models, analysis tools and compensation methods for the control of machines with friction. Automatica, 30(7):1083-1138, July 1994. 
[5] B. Bona and M. Indri. Friction compensation and robustness issues in force/position controlled manipulators. IEE Proceedings, Control Theory and Applications, 142(6):569-574, 1995.

[6] B. Bona, M. Indri, and N. Smaldone. Open system real time architecture and software design for robot control. In IEEE/ASME International Conference on Advanced Intelligent Mechatronics (AIM01), pages 349-354, Como (Italy), 2001.

[7] B. Bona, M. Indri, and N. Smaldone. An experimental setup for modelling, simulation and fast prototyping of mechanical arms. In IEEE Conference on ComputerAided Control Systems Design, Glasgow (UK), 2002.

[8] G. Calafiore, M. Indri, and B. Bona. Robot dynamic calibration: Optimal excitation trajectories and experimental parameter estimation. J. of Robotic Systems, 18(2):55-68, 2001.

[9] Y.-Y. Chen, P.-Y. Huang, and J.-Y-Yen. Frequencydomain identification algorithms for servo systems with friction. IEEE Trans. on Control Systems Technology, 10(5):654-665, September 2002.

[10] C. Canudas de Wit and P. Lischinsky. Adaptive friction compensation with partially known dynamic friction model. Int. J. of Adaptive Control and Signal Processing, 11:65-80, 1997.

[11] C. Canudas de Wit, P. Noël, A. Aubin, and B. Brogliato. Adaptive friction compensation in robot manipulators: Low velocities. Int. J. of Robotics Research, 10(3):189199, June 1991.

[12] C. Canudas de Wit, H. Olsson, K.J. Åström, and P. Lischinsky. A new model for control of systems with friction. IEEE Trans. on Automatic Control, 40(3):419425, March 1995.

[13] P. Dupont, B. Armstrong, and V. Hayward. Elasto-plastic friction model: contact compliance and stiction. In 2000 American Control Conference, pages 1072-1077, vol.2, 2000 .

[14] P.E. Dupont. Friction modeling in dynamic robot simulation. In IEEE International Conference on Robotics and Automation, pages 1370 -1376, vol. 2, 1990.

[15] R.H.A. Hensen, M.J.G. van de Molengraft, and M. Steinbuch. Frequency domain identification of dynamic friction model parameters. IEEE Trans. on Control Systems Technology, 10(2):191-196, March 2002.

[16] R.M. Hirschorn and G. Miller. Control of nonlinear systems with friction. IEEE Trans. on Control System Technology, 7(5):588-595, September 1999.
[17] Y.H. Kim and F.L. Lewis. Reinforcement adaptive learning neural-net-based friction compensation control for high speed and precision. IEEE Trans. on Control System Technology, 8(1):118-126, Jan. 2000.

[18] H. Olsson, K.J. Åström, C. Canudas de Wit, M. Gäfvert, and P. Lischinsky. Friction models and friction compensation. European Journal of Control, (4):176-195, 1998.

[19] L.R. Ray, A. Ramasubramanian, and J. Townsend. Adaptive friction compensation using extended kalman-bucy filter friction estimation. Control Engineering Practice, 9:169-179, 2001.

[20] J. Swevers, F. Al-Bender, C.G. Ganseman, and T. Prajogo. An integrated friction model structure with improved presliding behaviour for accurate friction compensation. IEEE Trans. on Automatic Control, 45(4):675686, April 2000. 